\title{
Éditorial Un objectif de santé publique : le vieillissement durable
}

$>$ Le vieillissement est généralement vu par les biologistes comme une diminution progressive de la fonctionnalité des organes, pouvant aboutir à la mort. II s'accompagne d'une augmentation de la susceptibilité de développer de nombreux cancers et maladies dégénératives pouvant toucher la quasi-totalité des organes (maladies neurodégénératives, cardio-vasculaires, métaboliques, pulmonaires et articulaires pour n'en citer que quelques unes). Savoir si le vieillissement est un processus programmé, comme l'est le développement, dont il pourrait constituer l'épilogue, ou simplement une accumulation aléatoire de dommages est une question polémique. En d'autres termes, existe-t-il une biologie propre du vieillissement ou simplement des situations physiopathologiques liées à la dégénérescence des organes et des systèmes? Cette question est fondamentale pour le traitement médical du vieillissement, car s'il existait une biologie propre du vieillissement, elle pourrait nous éclairer sur les déterminants biologiques communs à de nombreuses maladies. II serait alors possible d'intervenir sur ces déterminants pour prévenir simultanément l'apparition de nombreuses maladies dans la population générale. Cette approche révolutionnerait la médecine et notre société.

Les progrès récemment accomplis par la communauté biomédicale dans le domaine du vieillissement sont impressionnants et dessinent les contours de ce qui apparaît comme une biologie propre du vieillissement. Son concept « central » repose sur le fait que la cellule peut vieillir : on dit qu'elle devient sénescente et que cette sénescence est en grande partie responsable du vieillissement de l'organisme. Les travaux de recherche sur la sénescence cellulaire ont abouti à la description d'une série de dysfonctionnements moléculaires responsables du vieillissement: instabilité génomique, raccourcissement télomérique, altérations épigénétiques, perte de la protéostase, dérégulation du métabolisme énergétique, dysfonctionnement mitochondrial, épuisement des cellules souches et communication intercellulaire altérée [1]. Tous ces dysfonctionnements contribuent à l'accumulation des cellules sénescentes dans nos tissus, plaçant la sénescence au cœur de la biologie propre du vieillissement et suscitant une nouvelle stratégie pharmacologique, appelée sénothérapie, qui consiste à empêcher ou réverser l'accumulation de cellules sénescentes dans nos tissus pour prévenir et soigner les maladies du vieillissement. Cependant, à l'inverse, la sénescence peut être aussi bénéfique pour certaines étapes du développement, ainsi que pour la réparation tissulaire et la protection contre le cancer. Les futurs traitements sénolytiques devront donc respecter les «bonnes » cellules sénescentes et agir au bon moment et au bon endroit.

Le raccourcissement de l'ADN télomérique ${ }^{1}$ est le seul type de dysfonctionnement cellulaire observé lors du vieillissement qui soit également clairement programmé au cours du développement, avec une inhibition somatique de la télomérase en fin d'embryogenèse [2]. Le rôle clé de ce raccourcissement dans le vieillissement est d'ailleurs illustré par le fait que les patients porteurs de mutations dans les gènes de maintenance des télomères ont des télomères bien plus courts que ceux de la population générale, à âge égal, et souffrent d'un syndrome progeroïde (téloméropathies) ${ }^{22}$. De plus, dans la population générale, les télomères courts sont associés à un risque augmenté de mortalité, quelle que soit la cause de celleci.

Mais le raccourcissement de l'ADN télomérique n'est pas seulement la conséquence de l'incapacité de notre machinerie conventionnelle de réplication à dupliquer fidèlement les deux brins de la molécule d'ADN parentale; il est aussi le résultat d'un mécanisme spécifique, couplé à la réplication de nos extrémités de chromosome, qui entraîne la dégradation de l'extrémité du brin 5' d'une des deux chromatides sœurs. Ce mécanisme associe la fixation de l'exonucléase Apollo au télomère par l'intermédiaire de la sous-unité du complexe Shelterin appelée TRF2 (TTAGGG repeat binding factor 2$)^{1}$, elle-même fixée sur I'ADN télomérique double brin [3]. Il existe donc un mécanisme spécifique, sélectionné au cours de l'évolution, pour amplifier le raccourcissement de l'ADN télomérique lié à la réplication terminale, suggérant qu'un raccourcissement progressif peut conférer un avantage évolutif. Soulignons d'ailleurs qu'une patiente exprimant une protéine Apollo incapable d'interagir avec TRF2 présente une forme sévère

\footnotetext{
${ }^{1}$ Les extrémités naturelles de chromosomes, appelées télomères, sont formées d'une répétition en tandem de petits motifs d'ADN (5'-TTAGGG chez tous les vertébrés) qui sert de substrat à une transcriptase inverse, la télomérase, qui compense l'érosion réplicative de nos extrémités de chromosome, et de site de fixation pour le complexe protéique Shelterin qui stabilise les télomères en les empêchant d'être reconnus comme des cassures accidentelles.

${ }^{2}$ La dyskératose congénitale est le syndrome progéroïde de référence des téloméropathies avec ses formes sévères qui sont les syndromes de Hoyeraal-Hreidarsson et de Revesz. D'autres types de téloméropathies regroupent le syndrome de Coats plus, les anémies aplasiques et les fibroses pulmonaires idiopathiques.
} 
de téloméropathie [4]. De toutes ces données émerge donc la vision qu'un raccourcissement progressif des télomères, normalement contrôlé, peut être bénéfique au cours du développement embryonnaire, voire au cours de la vie adulte et $\mathrm{du}$ vieillissement. Cela pourrait s'expliquer par les propriétés onco-suppressives du raccourcissement de I'ADN télomérique. Cette question reste cependant largement sans réponse. II existe par ailleurs de plus en plus d'évidences montrant que les télomères et les mitochondries sont fonctionnellement liés, couplant le statut télomérique au métabolisme énergétique [5]. Une hypothèse intéressante qui en découle est que la taille des télomères serve de rhéostat au métabolisme au cours du développement et du vieillissement.

L'idée apparaît donc que le raccourcissement somatique des télomères est un programme génétique per se, liant développement et vieillissement. Mais combien de temps pouvons-nous vivre? Le taux de mortalité par année jusqu'à l'âge de 100 ans a fortement diminué depuis le milieu du XIX ${ }^{e}$ siècle dans les sociétés industrielles mais est demeuré constant au-delà, avec une espérance de vie maximale plafonnant autour de 120 ans (Jeanne Calment a atteint 122 ans) [6]. II apparaît donc que les avancées socio-économiques et médicales n'ont pas permis de faire reculer significativement le taux de mortalité annuel au-delà de 100 ans, suggérant l'existence d'une barrière biologique intrinsèque qui limite la vie humaine au-delà cet âge. La nature de cette barrière est inconnue. Que le programme télomère contribue à cette barrière est une hypothèse séduisante. Si la taille de nos télomères à la naissance et leur vitesse de raccourcissement dans les cellules somatiques sont en partie déterminées génétiquement, des situations de stress, y compris psychologiques, peuvent accélérer leur raccourcissement et contribuer à un vieillissement prématuré [2]. Par exemple, les adultes ayant été victimes de sévices dans leur enfance présentent des télomères plus courts que la taille normalement observée, et des signes de vieillissement prématuré. Ces résultats indiquent que, pour limiter l'apparition de maladies chroniques invalidantes dans la population âgée, pour obtenir un vieillissement que l'on pourrait qualifier de durable, il est essentiel de mieux comprendre les relations entre les processus biologiques du vieillissement, les histoires de vie, le comportement, le contexte socio-économique et l'environnement.
Un message donc à méditer par nos décideurs politiques. Un premier pas a été fait par l'Inserm dont un des programmes transversaux est consacré au vieillissement (consortium AGEMED «from aged cells to medical applications »), qui réunit plus de vingt équipes françaises représentant toutes les disciplines biologiques liées au vieillissement. Nous avons besoin maintenant d'élargir ce spectre disciplinaire par des approches holistiques et intégratives et de créer les conditions de coalitions internationales ambitieuses pour proposer à la société des solutions pour un vieillissement durable. $\diamond$

A public health goal: sustainable aging

\section{LIENS D'INTÉRÊT}

L'auteur déclare n'avoir aucun lien d'intérêt concernant les données publiées dans cet article.

\section{RÉFÉRENCES}

1. López-Otín C, Blasco MA, Partridge L, Serrano M, Kroemer G. The hallmarks of aging. Cell $2013 ; 153$ : 1194-217.

2. Blackburn $\varepsilon H$, , Epel ES. Telomeres and adversity: Too toxic to ignore. Nature 2012 ; $490: 169-71$.

3. Ye J, Lenain C, Bauwens S, et al. TRF2 and Apollo cooperate with topoisomerase 2alpha to protect human telomeres from replicative damage. Cell $2010 ; 142: 230-42$.

4. Touzot F, Callebaut I, Soulier J, et al. Function of Apollo (SNMIB) at telomere highlighted by a splice variant identified in a patient with Hoyeraal-Hreidarsson syndrome. Proc Natl Acad Sci USA 2010 ; 107 : 10097-102.

5. Robin J, Jacome Burbano MS, Peng H, et al. Mitochondrial function in skeletal myofibers is controlled by a TRF2-SIRT3 axis over lifetime. Aging Cell 2020 Jan 28 el3097. doi: 10.1111/acel.13097.

6. Burger 0 , Baudisch A, Vaupel JW. Human mortality improvement in evolutionary context. Proc Natl Acad Sci USA 2012 ; 109 : 18210-4.

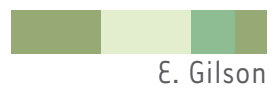

Université Côte-d'Azur, Inserm, CNRS Institut de Recherche sur le Cancer et le Vieillissement (Institute for Research on Cancer and Aging, Nice IRCAN) FHU OncoAge, Département de Génétique CHU, Nice, France. eric.gilson@unice.fr
TIRÉS À PART

ع. Gilson

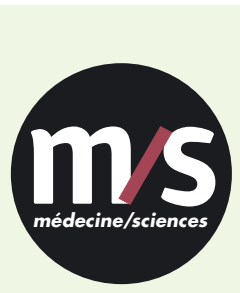

Tarifs d'abonnement $m / s-2020$

$>$ Grâce à $m / s$, vivez en direct les progrès des sciences biologiques et médicales

Abonnez-vous

à médecine/sciences

Bulletin d'abonnement page 294 dans ce numéro de $\mathrm{m} / \mathrm{s}$
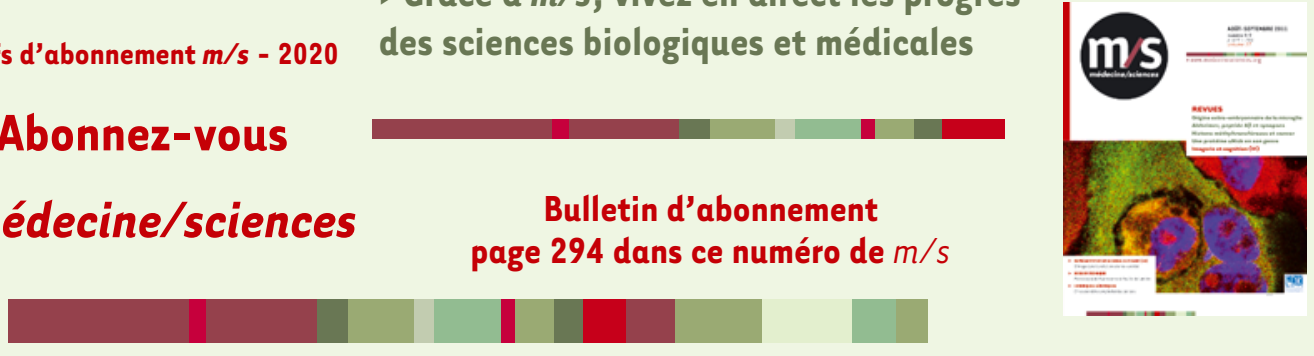